\title{
THE EFFECTS OF CUSTOMER EVALUATIONS OF CHANNEL INTEGRATION: DOES AN INTEGRATED MULTICHANNEL STRATEGY PAY OFF?
}

\author{
Hanna Schramm-Klein, University of Siegen, Germany \\ Gerhard Wagner, University of Siegen, Germany \\ Sascha Steinmann, University of Siegen, Germany \\ Dirk Morschett, University of Fribourg, Switzerland
}

\begin{abstract}
It has been common for retail companies to use multiple channels simultaneously (Rangaswamy and van Bruggen 2005). However, simultaneous use is only the first step in creating a customer-centric multichannel system that demands channel synergies rather than parallel retail formats (McGoldrick and Collins 2007). At issue is therefore not the degree of integration undertaken by a firm but rather the customer's perception of the extent of integration and how the channels allow a complementary and differentiated use of the multichannel system in purchase processes (Verhoef, Neslin, and Vroomen 2007). As such, cross-channel use of multichannel systems should offer customers more uses and new uses of retail channels than one-channel systems (Berman and Thelen 2004; Verhagen and van Dolen 2009).
\end{abstract}

In our study, an empirical survey using an online questionnaire was devised to target multichannel shoppers. We conducted an undirected addressing of participants on several independent internet pages and newsletters, to obtain a wide range of diverse target groups. Besides, a variety of sectors (apparel, books, groceries and cosmetics) were covered and multichannel systems with a range of differing characteristics were chosen. A sample of $\mathrm{N}=981$ customers who were familiar with the multichannel system of a retailer, i.e. at least three channel types (retail outlets, traditional catalogues and internet shops), were used in the data analysis.

We measured the evaluation of the individual channels of the multichannel system and the dimension of the evaluation of the perceived integration of the channels within the multichannel system separately for outlets, catalogues and internet shops. The overall evaluation of the individual retail channels was conceptualized as a formative construct based on customer's perception by calculating an index (weighted average of channel evaluations). We conceptualized the evaluation of perceived integration of the channels using the adequacy-importance model. Customer's likeability, i.e. the sympathy for a retailer (Keller 1998), and appreciation of a multichannel system were chosen as indicators of image (Dick and Basu 1994; de Ruyter, Wetzels, and Kleijnen 2001). Selnes (1998) suggests an one-dimensional global trust-assessment as the most reliable way to measure trust. Following this view, in this analysis trust was measured as a reflective approach using a direct assessment of customer's trust relating to each individual channel and the multichannel system as a whole (Sirdeshmukh, Singh, and Sabol 2002). Customer loyalty to the multichannel system was measured applying a formative approach. The intention to recommend a channel to family and friends was used as the indicator of loyalty (Andreassen and Lanseng 1997; Chaudhuri 1999). Customer's willingness of a differentiated use of a multichannel system, i.e. browsing and buying in different channels of a retailer's multichannel system was measured by using a formative approach. We employed one item to measure the participants' actual channel-using behavior, which includes the use/purchase frequency in each available channel, e.g. how often a customer uses a catalogue for buying or just for browsing. A second item measured the crosschannel usage in terms of using one channel to collect information but another one to purchase.

To test the hypotheses of our model we conducted Partial Least Squares (PLS) regression. The estimated path coefficients were all positive and significant. In particular, the findings show a relatively strong impact of customer's evaluation of individual channels on retailer's image $(\beta=.537)$ as well as a smaller effect on customer's trust $(\beta=.277)$ in the retailer. In contrary, customer's evaluation of perceived channel integration has a much stronger positive effect on trust $(\beta=.319)$ towards the retailer compared to the impact of this construct on the retailer's image $(\beta=.140)$. Overall, the results of our study indicate that linkages between retail channels positively affect customer loyalty (image $\rightarrow$ loyalty: $\beta=.402$; trust $\rightarrow$ loyalty: $\beta=.085$ ) and verify the importance of establishing a well-integrated multichannel system. These findings show also the high relevance of a customer oriented and well integrated multichannel strategy, as the positive effects of cognitive processes of trust and image formation have a direct positive effect on customer behavior (image $\rightarrow$ differentiated use of multichannel system: $\beta=.395$; trust $\rightarrow$ differentiated use of multichannel system: $\beta=.100$ ). Generally, the results of our study indicate that a retailer should have a strong focus on customers' perception and evaluation of individual channels as well as on the perception of channel integration for an effective and efficient multichannel system.

References available upon request 